\title{
Absorbance response of graphene oxide coated on tapered multimode optical fiber towards liquid ethanol
}

S. H. Girei

gireisaad3@gmail.com

\section{A. A. Shabaneh \\ H. M. Lim \\ N. M. Huang}

M. A. Mahdi

M. H. Yaacob
Wireless and Photonics Network Research Center, Universiti Putra Malaysia, 43400 UPM Serdang, Selangor, Malaysia

Wireless and Photonics Network Research Center, Universiti Putra Malaysia, 43400 UPM Serdang, Selangor, Malaysia

Wireless and Photonics Network Research Center, Universiti Putra Malaysia, 43400 UPM Serdang, Selangor, Malaysia

Low Dimension Research Centre, Physics Department, Faculty of Science, University of Malaya, 50603 Kuala Lumpur, Malaysia

Wireless and Photonic Network Research Center, Universiti Putra Malaysia, Malaysia, 43400 UPM Serdang, Selangor, Malaysia

Wireless and Photonic Network Research Center, Universiti Putra Malaysia, Malaysia, 43400 UPM Serdang, Selangor, Malaysia

The investigation of graphene oxide (GO) for sensing applications is attractive due to its nanoscale structure and its sensing properties has yet to be fully understood. In this paper, optical response of GO coated optical fiber sensor towards ethanol is described. CO was coated onto a multimode tapered optical fiber by drop-casting technique. The coated fiber was exposed to $5-40 \%$ of ethanol in water. The films were characterized with field emission scanning electron microscope, ultraviolet-visible spectroscopy and Raman spectroscopy. The sensing is based on changes following the absorbance of the $\mathrm{GO}$ coated optical fiber upon exposure to ethanol. The developed sensor shows fast response and recovery with duration of 22 and $20 \mathrm{~s}$, respectively. The sensor also displays high repeatability and reversibility.

[DOI: http://dx.doi.org/10.2971/jeos.2015.15019]

Keywords: Absorbance, ethanol, graphene oxide, optical fiber sensor, tapered fiber

\section{INTRODUCTION}

Volatile organic compounds (VOC) such as ethanol are commonly used in the commercial applications especially in chemical and food industries [1]. Ethanol is flammable liquid which can cause eye and respiratory tract irritation when in contact with human [2]. Therefore, it is important to monitor ethanol accurately in the industrial processes. Common ethanol sensors were developed by using electrical transducers. For instance, Weng et. al [3] have developed an amperometry ethanol sensor with sputtered $\mathrm{Ni} / \mathrm{Pt} / \mathrm{Ti}$ as sensing layer. The sensitivity of the sensor was approximately $3.08 \mu \mathrm{A} \mu \mathrm{M}^{-1} \mathrm{~cm}^{-2}$. Bairiu et al. [4] also fabricated an amperometry sensor based on silicon nanowires with palladium-nickel as the working electrode. The sensor had sensitivity of $7.8 \mathrm{mAmM}^{-1} \mathrm{~cm}^{-2}$ with detection limit of $6 \mu \mathrm{M}$. Khan et al. [5] have developed an aqueous ethanol sensor with improved sensitivity. Their sensor was based on poly propylene carbonate (PPC) synthesized using sol-gel method. The electrical based sensors are relatively low cost and more sensitive as compared to the optical based sensors [6]. However, there is a rapid and growing interest in the field of optical fiber sensors. Owing to some attractive and unique properties such as immunity to electromagnetic interference, resistance to high temperature, small size as well as lightweight, optical fiber is a suitable candidate for the detection of VOCs [7].

Recently, studies based on tapered optical fiber have received much attention as compared to conventional fibers [8]. Tapered optical fiber was found to be more sensitive as compared to the conventional fiber due to the manner of light propagation in the tapered optical fiber core. A fraction of the optical power was found to propagate outside the tapered optical fiber core [9]. In other words, the evanescent field of the tapered fiber goes beyond the fiber physical boundary. Coating the tapered region with sensing layer allows the interaction of the layer with the evanescent field. When the analyte interacts with the sensing layer and changes the layer properties, the evanescent field will also be affected [10]. The sensing layer, which can be in the form of thin film, is designed to be permeable and sensitive to a specific analyte [11]. Previously, semiconducting metal oxides [12, 13], carbon nanotubes [14] and polymeric [15] materials have been widely used as sensitive layer in various optical fiber sensing applications such as chemical and bio sensing. Optical sensor integrates with single wall carbon nanotubes [16] and silicon quantum dot [17] for ethanol vapor sensing have been reported in literatures. Fiber optic sensors for detection of low level water content in ethanol based on surface plasmon resonance [18] and 
micro-bending [19] have also been reported. In recent years, graphene oxide (GO) has attracted much attention in the field of sensing [20]. This is as a result of its excellent electrical, mechanical and thermal properties [21]. The hydrophilic nature of GO allows it to be uniformly deposited onto different substrates [22]. This is a prerequisite in applications such as microelectronics and sensors. However, only very few studies focused on the tapered optical fiber coated with GO for ethanol sensing applications [23].

In this paper, GO coated on tapered multimode optical fiber sensor for sensing of different concentrations of ethanol in water is described. The sensitivity and the optical response of the GO coated and bare tapered fiber sensor towards ethanol are compared and presented.

\section{EXPERIMENTAL DETAILS}

\subsection{Tapered optical fiber fabrication}

Standard multimode step index silica fibres with core and cladding diameter of 62.5 and $125 \mu \mathrm{m}$, respectively, are used in this experiment. The tapering of the optical fiber is done using Vytran glass processing workstation (GPX 3000). The ends of the fiber are fixed to the fiber holding block with the section to be tapered placed above the filament heater. Prior to that, the polymeric coating of the optical fiber is removed mechanically for length of about $5 \mathrm{~cm}$ and cleaned with acetone. The working principle of the machine is based on heating the optical fiber with filament heater while tensile force is applied by the holding blocks. The cross sectional area of the optical fiber reduces accordingly as it is elongated. Proprietary software is used to ascertain taper parameters. Tapered optical fiber with waist length of $20 \mathrm{~mm}$ and waist diameter of $40 \mu \mathrm{m}$ with transition length of $2 \mathrm{~mm}$ is fabricated.

\subsection{G0 thin film preparation}

GO solution is prepared using the simplified Hummers method [24, 25]. GO solution with concentration of $1 \mathrm{mg} / \mathrm{ml}$ is drop-casted on the tapered area of the multimode tapered optical fiber. Subsequently, the tapered optical fiber is annealed at $70^{\circ} \mathrm{C}$ for an hour and left overnight at room temperature. GO thin film is characterised using field emission scanning electron microscopy (FESEM), ultraviolet-visible spectroscopy and Raman spectroscopy.

\subsection{Experimental setup}

The optical sensing setup is shown in Figure 1. A tungstenhalogen lamp (Ocean Optics HL2000) with wavelength range of $360-2400 \mathrm{~nm}$ is used as a light source. A spectrometer (Ocean Optics USB4000) with spectral range of $200-1100 \mathrm{~nm}$ is used as the light detector and spectrum analyzer. The spectrometer is connected to computer via USB port and data are analyzed using SpectraSuite software. A customised chamber is deployed to expose the developed sensor to ethanol with different concentrations. Ethanol with different concentrations is prepared by mixing appropriate amounts of deionised water and ethanol. The volume percentage of ethanol in distilled water was varied from $5 \%$ to $40 \%$.

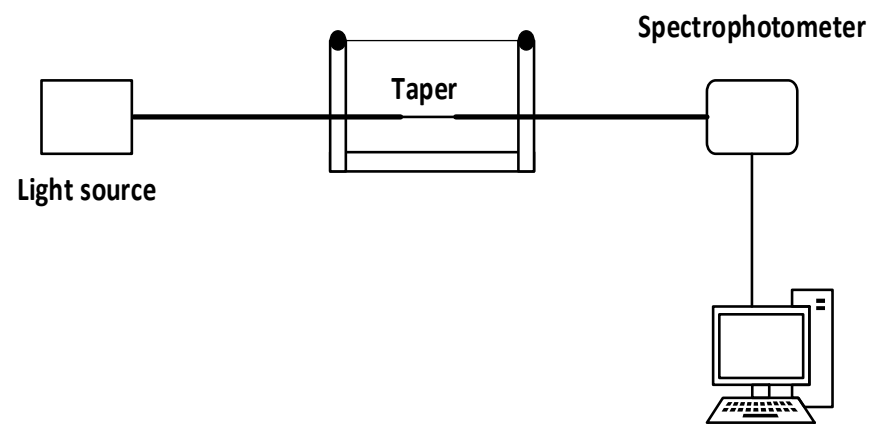

PC

FIG. 1 Optical sensing setup.

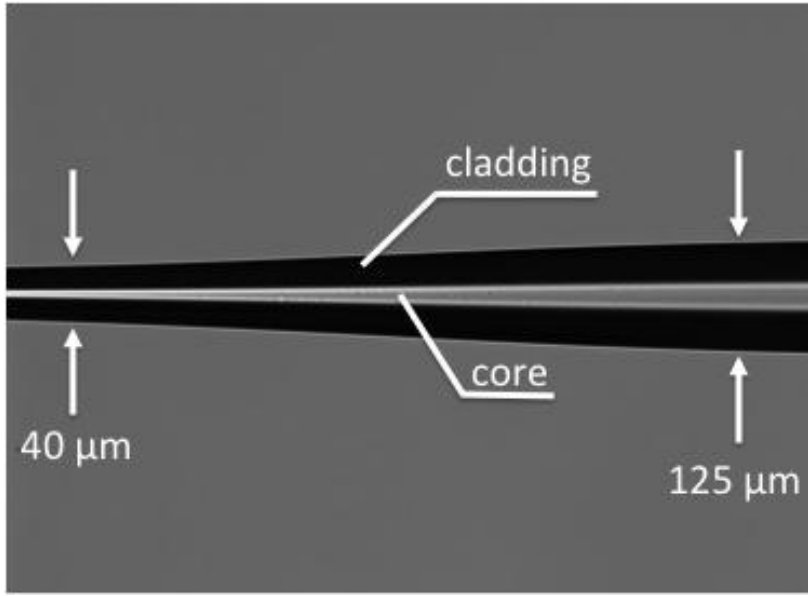

FIC. 2 CCD image of a segment of the tapered fiber with waist diameter of $40 \mu \mathrm{m}$ and its Original diameter of $125 \mu \mathrm{m}$.

\section{RESULTS AND DISCUSSION}

\subsection{Optical fiber and GO characterization}

Figure 2 shows optical fiber $(125 \mu \mathrm{m})$ that is tapered to $40 \mu \mathrm{m}$ waist diameter. After tapering, the fundamental mode of the light signals previously propagating in the core is now propagating in the cladding of the tapered optical fiber. The sufficiently long taper transition creates an adiabatic criterion, which minimizes the loss of propagating signals [26]. The adiabatic criterion result to the transformation of the fundamental mode to the cladding mode after tapering [27]. This transformation increases the penetration depth of the evanescent field. The throughput power of the optical fiber recorded an average loss of $0.65 \mathrm{~dB}$ after tapering.

Figure 3 shows the scanning electron microscopy (SEM) image of the synthesised GO. The image reveals the typical synthesised GO is in sheet structures with lateral dimension around 20-50 $\mu \mathrm{m}$. The thickness of the GO sheets was measured to be approximately $1 \mathrm{~nm}$ and has been reported elsewhere [28]. The high surface area provides by the GO nanosheets can improve the ethanol molecules interaction with the sensing layer. Consequently, the absorbance response of the GO-coated optical fiber may improve significantly.

Figure 4(a) illustrate the SEM image of an uncoated tapered optical fiber while Figure 4(b) shows the image of the tapered optical fiber coated with GO. The images clearly show the smooth surface of the tapered fiber was transformed into rough surface upon GO deposition. The GO thin film exhib- 


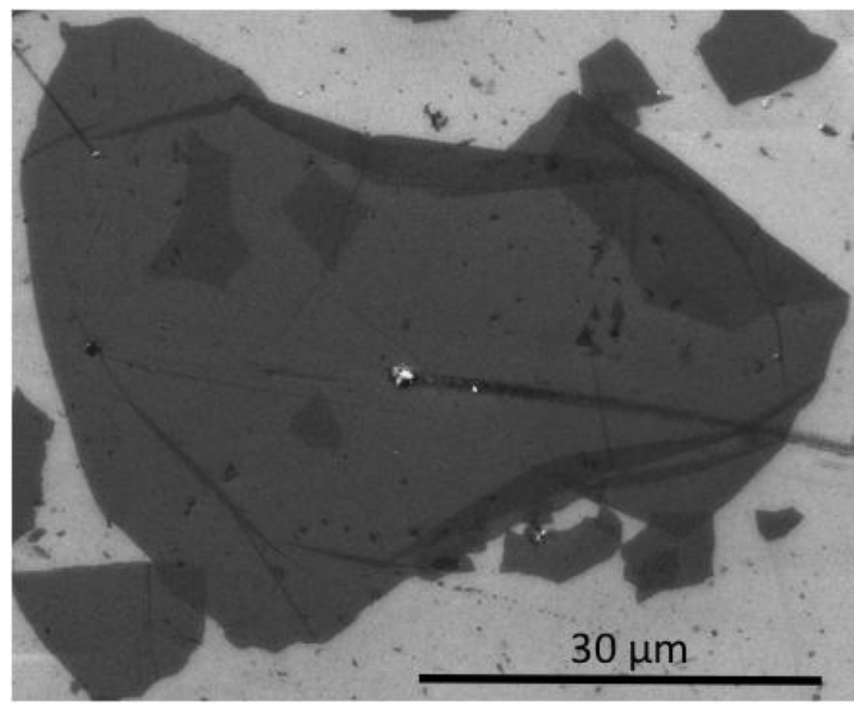

FIG. 3 SEM image of a single GO nanosheet.

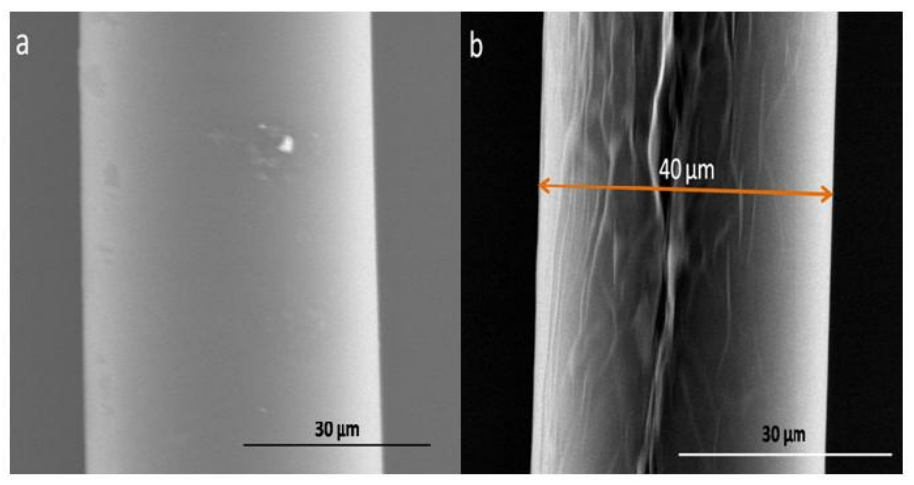

FIG. 4 SEM images of tapered optical fiber in the (a) absence and (b) presence of CO.

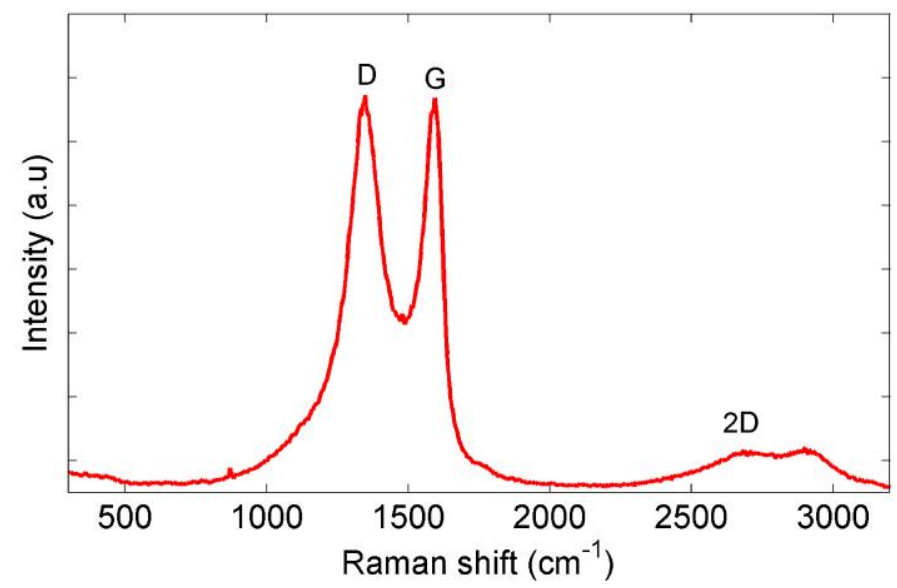

FIG. 5 Raman spectrum of 60 on glass substrate.

ited a corrugated and wrinkled structure with homogeneous morphology. The drop casting method used was able to produce a homogeneous deposition of GO on tapered optical fiber.

Figure 5 depicts the Raman spectrum of GO. It features the typical $\mathrm{D}$ band $\left(1348 \mathrm{~cm}^{-1}\right)$ which is assigned to local defects and disorder of graphene that is caused by the attachment of hydroxyl and epoxide groups on the basal plane and edges, and the $\mathrm{G}$ band $\left(1590 \mathrm{~cm}^{-1}\right)$ which corresponds to first order scattering of E2g mode [29, 30]. GO Raman spectra also shows $2 \mathrm{D}$ band around $2685 \mathrm{~cm}^{-1}$, this band is the second order of $\mathrm{D}$ band [31].
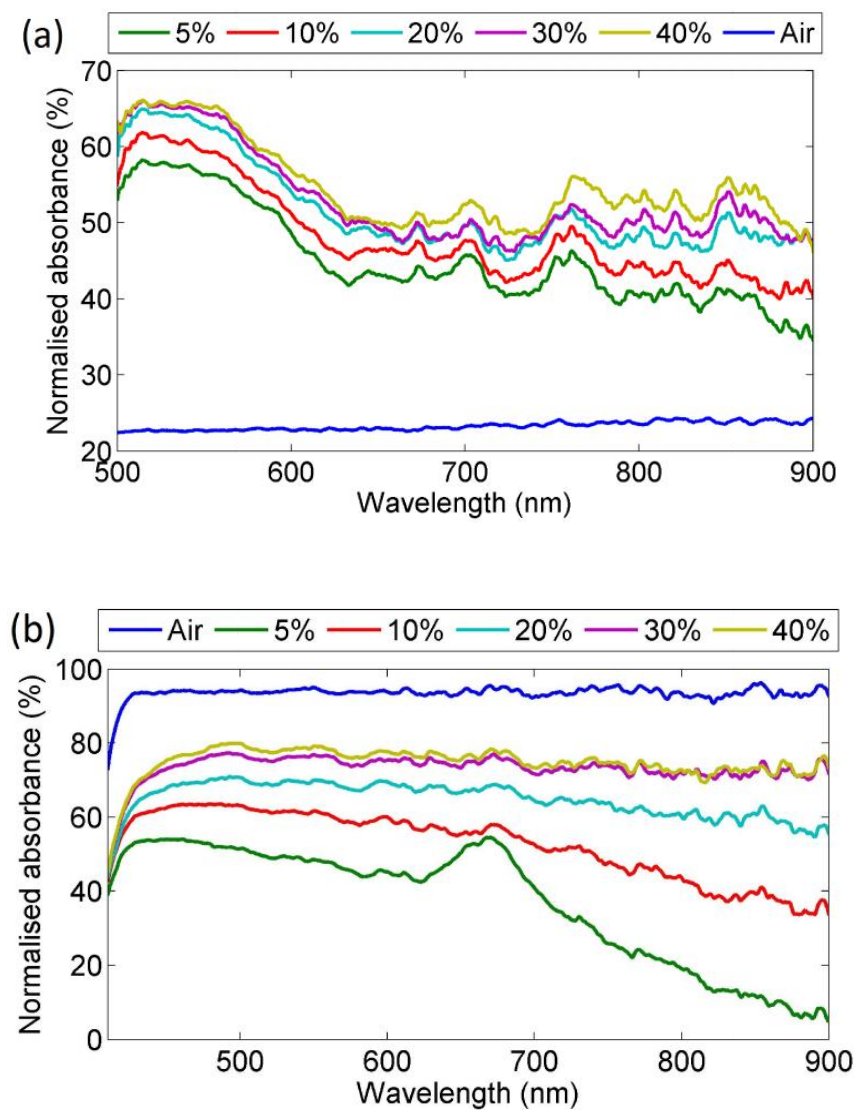

FIG. 6 Absorbance spectra of (a) uncoated tapered optical fiber and (b) G0 coated tapered optical fiber exposed to ethanol with different concentrations.

\subsection{Ethanol sensing performance}

The interaction between the analyte and the sensing layer was studied by monitoring the absorbance spectrum of the developed sensor exposed to ethanol with different concentrations at room temperature. The change in absorbance was monitored in the wavelength range of $400 \mathrm{~nm}-900 \mathrm{~nm}$ and for $5 \%-40 \%$ ethanol in water. The absorbance spectra of the developed sensor towards ethanol are displayed in Figure 6. It was found that the absorbance response increases with the increase of ethanol concentrations for the uncoated fiber (Figure 6(a)). Since the silica fiber is inert, the response is concluded due to the change in the refractive index for the different ethanol concentrations. The tapering of the optical fiber significantly improves the fiber sensitivity [10] and thus, distinct absorbance responses. For the GO coated fiber (Figure 6(b)), it was observed that the sensors initial absorbance in air is high (90\%) due to the GO layer coating. When exposed to ethanol, the absorbance response increases with the increase in the ethanol concentrations. The response is larger in the GO coated fiber as compared to the response in the uncoated fiber. This is possibly due to the high surface area of the GO layer increases the ethanol molecules interaction with the layer and thus, significantly changes the evanescent field. In addition, GO is known to possess excellent physicochemical properties that can enhance the sensing response towards ethanol [32]. However, the magnitude of the response reduces with the increase of the concentrations. The trend was observed across the visible wavelength range.

Taking an example at $600 \mathrm{~nm}$ wavelengths, the normalized absorbance was found to be $45 \%, 60 \%, 70 \%, 75 \%$ and $78 \%$ upon 
exposure to ethanol with $5 \%, 10 \%, 20 \%, 30 \%$ and $40 \%$ concentrations, respectively. There is a small increase in the absorbance from $30 \%$ to $40 \%$ of ethanol concentration in water, which indicates saturation in the sensor response due to high ethanol concentrations. The peak around $660 \mathrm{~nm}$ in Fig 6a was identified to be induced by absorption of water [33]. The peak diminishes as the concentration of ethanol increases because the surface of the GO coated fiber gradually interacts more with ethanol instead of water. This has caused the wavelength to be absorbed dominantly by ethanol instead of water.

Figure 7 exhibits the dynamic responses of the tapered fiber sensor (a) without GO and (b) with GO towards different concentrations of ethanol in water at room temperature. Both sensors show distinct sensitivity towards different concentrations of ethanol. The magnitude of response reduces with the increase in the ethanol concentrations due to saturation of ethanol on the GO coated fiber, which is in good agreement with the response in Figure 6. A maximum absorbance decrease of $78 \%$ can be observed when the developed sensor was exposed to ethanol with $5 \%$ concentrations. Compared to the response of the uncoated multimode tapered fiber (Figure $7(\mathrm{a}))$, this is a significant improvement in the sensitivity by a factor of 3. The GO coated fiber also exhibited stable baseline when exposed to the different ethanol concentrations. The stability and excellent response and recovery of the GO coated sensor might be as a result of the high GO surface area due to the wrinkles and the presence of larger interlayer space in the GO films which improves ethanol-water mixture desorption [34]. The dynamic response of the sensor coated with GO exhibit a negative variation of the absorbance to its counterpart of the uncoated optical fiber. The response and recovery times of the tapered optical fiber sensor coated with GO for $5 \%$ ethanol in water are $22 \mathrm{~s}$ and $20 \mathrm{~s}$, respectively.

The repeatability and reversibility of the sensor is presented in Figure 8 . The sensor was immersed to three consecutive cycles in the presence of $5 \%$ ethanol concentration and in air at room temperature. The stable result implies that the GO adhered well onto the surface of the optical fiber. The good compatibility of the GO and optical fiber interface is contributed by electrostatic interaction between the oxide functional groups of GO and the siliceous material of the optical fiber.

\section{CONCLUSIONS}

In this work, a simple multimode tapered fiber sensor based on GO was developed. The sensor exhibits repetitive and reversible behaviour with response and recovery times in the realm of $20 \mathrm{~s}$. The maximum response of the developed GO coated sensor increased, by a factor of 3.0 as compared to the bare sensor. The modified sensor is a promising detector for real-time ethanol sensing applications in various industries.

\section{ACKN OWLEDGMENTS}

The authors would like to acknowledge Universiti Putra Malaysia for the project fund under Research University Grant Scheme (RUGS) No. 05-02-12-2015RU.
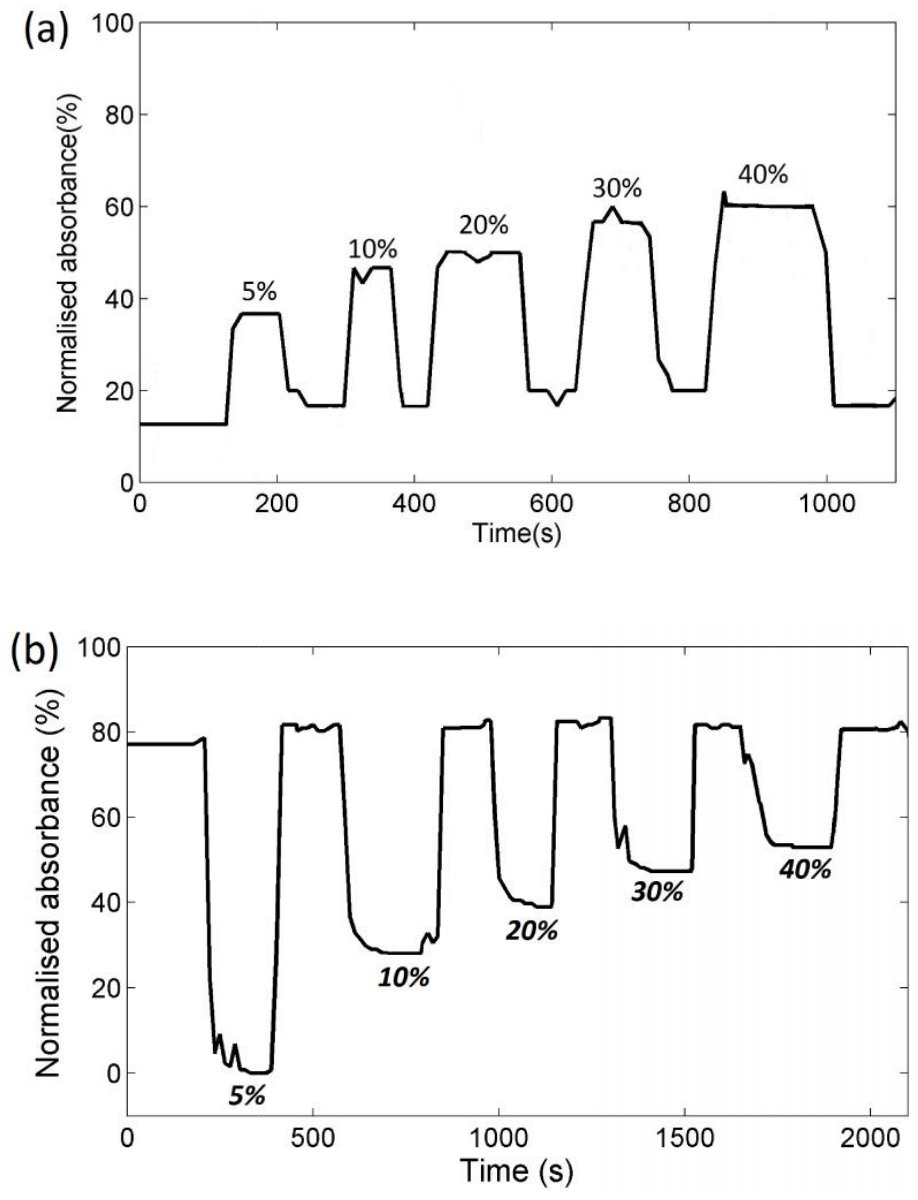

FIG. 7 Dynamic response of tapered optical fiber towards 5-40(b) with G0.

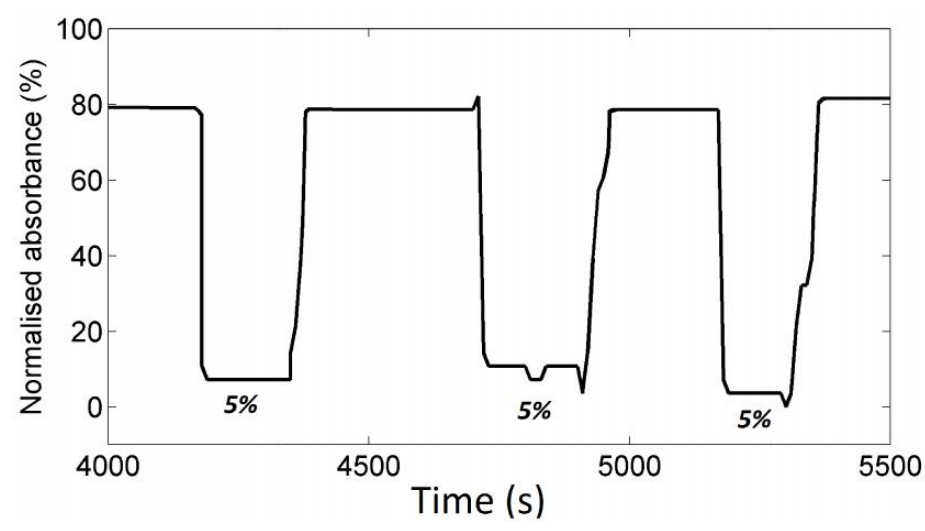

FIG. 8 Repeatability and reversibility test towards $5 \%$ ethanol.

\section{References}

[1] C. Elosua, I. Matias, C. Bariain, and F. Arregui, "Volatile organic compound optical fiber sensors: A review," Sensors 6, 1440-1465 (2006).

[2] http://fscimage.fishersci.com/msds/89308.htm.

[3] Y. Weng, J. Rick, and T. Chou, "A sputtered thin film of nanostructured $\mathrm{Ni} / \mathrm{Pt} / \mathrm{Ti}$ on $\mathrm{Al}_{2} \mathrm{O}_{3}$ substrate for ethanol sensing," Biosens. Bioelectron. 20, 41-51 (2004).

[4] B. Tao, J. Zhang, S. Hui, and L. Wan, "An amperometric ethanol sensor based on a Pd-Ni/SiNWs electrode," Sensor Actuat. BChem. 142, 298-303, (2009).

[5] S. Khan, M. Rahman, and K. Akhtar, "Novel and sensitive ethanol chemi-sensor based on nanohybrid materials," Int J Electrochem. Sc. 7, 4030-4038 (2012). 
[6] B. Lee, "Review of the present status of optical fiber sensors," Opt. Fiber Technol. 9, 57-79 (2003).

[7] M. Yasin, S. W. Harun, and H. Arof, Fiber optics sensors (Croatia: InTech, Rijeka, 2012).

[8] H. Y. Lin, C. H. Huang, G. L. Cheng, N. K. Chen, and H. C. Chui, "Tapered optical fiber sensor based on localized surface plasmon resonance.," Opt. Express 20, 21693-701 (2012).

[9] S. Guo and S. Albin, "Transmission property and evanescent wave absorption of cladded multimode fiber tapers," Opt. Express 11, 215-223 (2003).

[10] J. Villatoro, D. Monzón-Hernández, and D. Luna-Moreno, “Inline optical fiber sensors based on cladded multimode tapered fibers.," Appl. Opt. 43 5933-5938 (2004).

[11] G. Brambilla, "Optical fibre nanotaper sensors," Opt. Fiber Technol. 16, 331-342 (2010).

[12] J. Z. Ou, M. H. Yaacob, J. L. Campbell, M. Breedon, K. Kalantarzadeh, and W. Wlodarski, "H sensing performance of optical fiber coated with nano-platelet W03 film," Sensors Actuat. B-Chem. 166-167, 1-6 (2012).

[13] B. Renganathan, D. Sastikumar, G. Gobi, N. Rajeswari Yogamalar, and A. Chandra Bose, "Nanocrystalline Zno coated fibser optic sensor for ammonia gas detection," Opt. Laser Technol. 43, 1398-1404 (2011).

[14] S. Manivannan, A. M. Saranya, B. Renganathan, D. Sastikumar, G. Cobi, and K. C. Park, "Single-walled carbon nanotubes wrapped poly-methyl methacrylate fiber optic sensor for ammonia, ethanol and methanol vapors at room temperature," Sensors Actuat. BChem. 171-172, 634-638 (2012).

[15] J. Yuan and M. El-Sherif, "Fiber-optic chemical sensor using polyaniline as modified cladding material," IEEE Sens. J. 3, 5-12 (2003).

[16] M. Consales, A. Crescitelli, M. Penza, P. Aversa, P. D. Veneri, M. Giordano, and A. Cusano, "SWCNT nano-composite optical sensors for VOC and gas trace detection," Sensors Actuat. B-Chem. 138, 351-361, (2009).

[17] Z. . Zhang, R. Lockwood, J. G. . Veinot, and A. Meldrum, “Detection of ethanol and water vapor with silicon quantum dots coupled to an optical fiber," Sensors Actuat. B-Chem. 181, 523-528 (2013).

[18] S. K. Srivastava, R. Verma, and B. D. Gupta, "Surface plasmon resonance based fiber optic sensor for the detection of low water content in ethanol," Sensors Actuat. B-Chem. 153, 194-198 (2011).

[19] F. B. Xiong and D. Sisler, "Determination of low-level water content in ethanol by fiber-optic evanescent absorption sensor," 0pt. Commun. 283, 1326-1330 (2010).

[20] S. Prezioso, F. Perrozzi, L. Giancaterini, C. Cantalini, E. Treossi, V. Palermo, M. Nardone, et al., "Graphene oxide as a practical solution to high sensitivity gas sensing," J. Phys. Chem. C 117, 10683-10690 (2013).
[21] V. Singh, D. Joung, L. Zhai, and S. Das, "Graphene based materials: past, present and future," Prog. Mater. Sci. 56, 1178-1271 (2011).

[22] F. A. Chowdhury, T. Morisaki, J. Otsuki, and M. Sahabul Alam, "Annealing effect on the optoelectronic properties of graphene oxide thin films," Appl. Nanosci. 3, 477-483 (2012).

[23] A. Aziz, H. N. Lim, S. H. Girei, M. H. Yaacob, M. A. Mahdi, N. M. Huang, and A. Pandikumar, "Silver/graphene nanocomposite-modified optical fiber sensor platform for ethanol detection in water medium," Sensors Actuat. B-Chem. 206, 119-125 (2015).

[24] W. H. Jr and R. Offeman, "Preparation of graphitic oxide," J. Am. Chem. 208, 1339 (1958).

[25] H. N. Lim, N. M. Huang, S. S. Lim, I. Harrison, and C. H. Chia, “Fabrication and characterization of graphene hydrogel via hydrothermal approach as a scaffold for preliminary study of cell growth.," Int. J. Nanomed. 6, 1817-23, (2011).

[26] S. W. Harun, K. S. Lim, C. K. Tio, K. Dimyati, and H. Ahmad, "Theoretical analysis and fabrication of tapered fiber," Opt. Int. J. Light Electron 0pt. 124, 538-543 (2013).

[27] T. Birks and Y. Li, "The shape of fiber tapers," Light. Technol. J. 10, 432-438 (1992).

[28] N. F. Lokman, A. A. A. Bakar, F. Suja, H. Abdullah, W. B. W. A. Rahman, N.-M. Huang, and M. H. Yaacob, "Highly sensitive SPR response of $\mathrm{Au} / \mathrm{chitosan} / \mathrm{graphene}$ oxide nanostructured thin films toward Pb (II) ions," Sensors Actuat. B-Chem. 195, 459-466 (2014).

[29] D. Yang, A. Velamakanni, G. Bozoklu, S. Park, M. Stoller, R. D. Piner, S. Stankovich, I. Jung, et al., "Chemical analysis of graphene oxide films after heat and chemical treatments by $X$-ray photoelectron and Micro-Raman spectroscopy," Carbon N. Y. 47, 145-152 (2009).

[30] L. Nikiel and P. W. Jagodzinski, "Raman spectroscopic characterization of graphites: A re-evaluation of spectra/ structure correlation," Carbon N. Y. 31, 1313-1317 (1993).

[31] S. Gurunathan, J. W. Han, V. Eppakayala, and J.-H. Kim, "Biocompatibility of microbially reduced graphene oxide in primary mouse embryonic fibroblast cells.," Colloids Surf. B. Biointerfaces 105, 5866 (2013).

[32] Y. Chang, Y. Yao, B. Wang, H. Luo, T. Li, and L. Zhi, "Reduced graphene oxide mediated $\mathrm{SnO}_{2}$ nanocrystals for enhanced gassensing properties," J. Mater. Sci. Technol. 29, 157-160 (2013).

[33] V. S. Langford, A. J. Mckinley, and T. I. Quickenden, "Temperature dependence of the visible-near-infrared absorption spectrum of liquid water," J. Phys. Chem. A 105, 8916-8921 (2001).

[34] J. I. Paredes, S. Villar-Rodil, a Martínez-Alonso, and J. M. D. Tascón, "Graphene oxide dispersions in organic solvents.," Langmuir 24, 10560-4 (2008). 